\title{
Interpretation on Deep Impact results: Radial distribution of ejecta and the size distribution of large-sized grains
}

\author{
T. Kadono ${ }^{1}$, S. Sugita ${ }^{2}$, T. Ootsubo ${ }^{3}$, S. Sako ${ }^{4}$, T. Miyata ${ }^{4}$, R. Furusho ${ }^{5}$, M. Honda ${ }^{6}$, H. Kawakita ${ }^{7}$, and J. Watanabe \\ ${ }^{1}$ Institute of Laser Engineering, Osaka University, Japan \\ ${ }^{2}$ Department of Complexity Science and Engineering, the University of Tokyo, Japan \\ ${ }^{3}$ Institute of Space and Astronautical Science, Japan Aerospace Exploration Agency, Japan \\ ${ }^{4}$ Institute of Astronomy, the University of Tokyo, Japan \\ ${ }^{5}$ National Astronomical Observatory of Japan, Japan \\ ${ }^{6}$ Kanagawa University, Japan \\ ${ }^{7}$ Department of Physics, Kyoto Sangyo University, Japan
}

(Received July 31, 2008; Revised December 3, 2008; Accepted December 10, 2008; Online published February 12, 2010)

\begin{abstract}
Several observations of dust grains ejected from the comet 9P/Tempel 1 by the Deep Impact event strongly suggest that the evaporation and expansion of volatiles occurred and that the vapor accelerated some dust grains. When grains are accelerated by gas, size sorting should occur, that is, larger grains tend to stay closer to the nucleus, while smaller grains is pushed farther away. This means that the light at each distance is emitted from identical-sized grains. Hence, we can estimate the size distribution of grains based on the flux as a function of the distance from the nucleus. A simple evaluation indicates that the size distribution of grains with a size larger than $\sim 10 \mu \mathrm{m}$ is expressed as a power law and the index is $\sim-4$. This is expected to be an alternative method to estimate the size distribution of grains, though detailed analyses and numerical simulations should be necessary to evaluate the error of this method.
\end{abstract}

Key words: Jupiter family comets, Deep Impact, ejecta, size distribution, MIR observation, SUBARU telescope.

\section{Introduction}

The impactor launched from the Deep-Impact (DI) spacecraft collided with the comet 9P/Tempel 1 at 2005/7/4 (UT) (A'Hearn et al., 2005). The large amount of cometary materials was ejected. We conducted mid-infrared (mid-IR) observations before and after its collision using COMICS (the Cooled Mid-IR Camera and Spectrometer), mounted on the Subaru Telescope, and both imaging and spectroscopic measurements were performed with three band-pass filters in the $N$-band with central wavelengths of 8.8, 10.5, and $12.4 \mu \mathrm{m}$ (Sugita et al., 2005; Kadono et al., 2007; Ootsubo et al., 2007). The observational data indicate that large-scale dust plume ejected by the impact contained a large mass of $\sim 10^{6} \mathrm{~kg}$ of solid dust grains and formed two wings approximately \pm 45 degrees from the symmetric center (Sugita et al., 2005). These facts are consistent with the gravity-controlled nature of the DI-induced impact cratering. Also, the image data indicate that there are two regions in the plume. In the outer region, which is far from the nucleus and contains dust grains with higher ejection velocities, the slope of the blackbody continuum is flat and the intensity of $10.5 \mu \mathrm{m}$ silicate feature is small relative to the continuum, and in the inner region, the slope is steep (the intensity at longer wavelength is large) and the intensity of $10.5 \mu \mathrm{m}$ is relatively large. We can quanti-

Copyright (C) The Society of Geomagnetism and Earth, Planetary and Space Sciences (SGEPSS); The Seismological Society of Japan; The Volcanological Society of Japan; The Geodetic Society of Japan; The Japanese Society for Planetary Sciences; TERRAPUB.

doi:10.5047/eps.2008.12.005 tatively understand this result using a simple dust model (e.g., Ootsubo et al., 2007), and this result and the crater formation mechanism (e.g., Melosh, 1989) indicate that the comet 9P/Tempel 1 has a surface layer with a large fraction of small (sub-micron sized) carbonaceous grains whose thickness is $\sim 1 \mathrm{~m}$ and a subsurface region consisting of a large fraction of sub-micron sized silicate grains (Kadono et al., 2007).

Spectral analyses using the data at the mid-infrared wavelength do not clearly indicate the size distribution at a size larger than $\sim 10 \mu \mathrm{m}$ because large ejecta grains are not optically active. However, their contribution to the total mass of DI ejecta plume is very large. Thus, estimation of the size distribution of the large ejecta grains is of extremely high importance. Consequently, the method to obtain the size distribution at larger sizes is necessary for precise estimation of ejecta mass. Size distribution of large grains can be estimated by modeling the effect of radiation pressure on the grains based on the images of dust clouds (e.g., Jorda et al., 2007). In this paper, we propose an alternative method to estimate the size distribution at a size larger than $\sim 10 \mu \mathrm{m}$ using the observational results of the DI event, taking advantage of the fact that the ejecta release process from the DI impact was practically instantaneous unlike normal activities.

\section{Observational Results}

We denote the flux at each wavelength in the $N$-band as $I_{8.8}, I_{10.5}$, and $I_{12.4}$, respectively. For all the results, the flux on July 2 (i.e., two days before the collision) is subtracted 


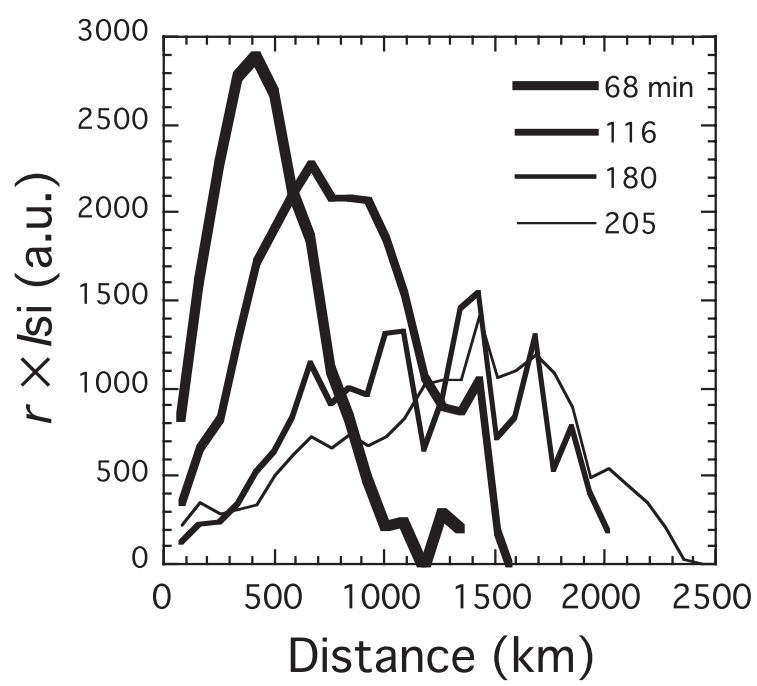

Fig. 1. Radial profiles of $I_{\mathrm{si}}\left(=I_{10.5}-\left(I_{8.8}+I_{12.4}\right) / 2\right)$ multiplied by $r$ as a function of time at $\sim 1,2,3$, and 3.5 hours after the impact.

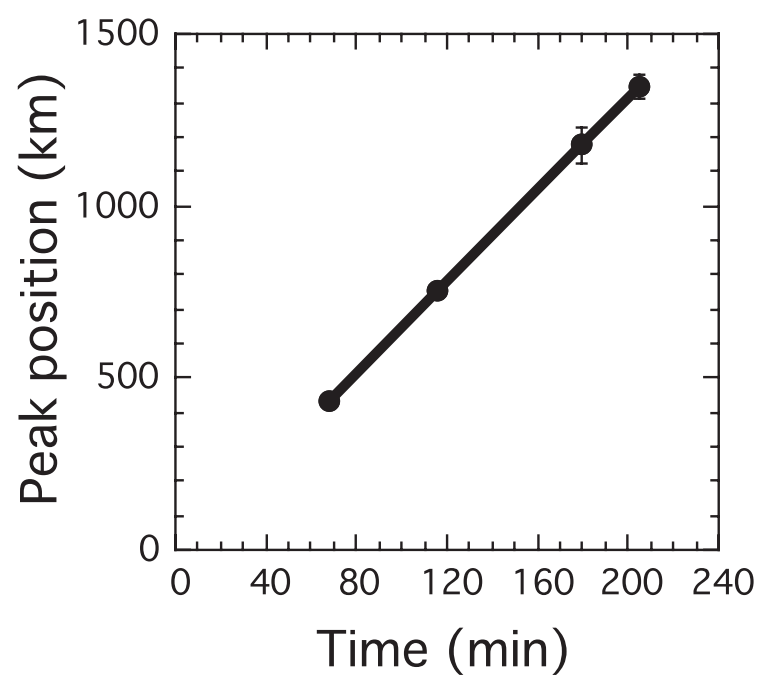

Fig. 2. The positions of the peaks of $r I_{\mathrm{si}}$ shown in Fig. 1 as a function of time. It looks that the peaks move outward. Linear fitting indicates that the expansion velocity is $111 \mathrm{~m} / \mathrm{s}$.

as a background at each wavelength.

First, we consider $I_{\mathrm{si}}$, which is defined as $I_{10.5}-I_{\text {cont }}$, where $I_{\text {cont }}$ is $\left(I_{8.8}+I_{12.4}\right) / 2$. This intensity of the 10 $\mu \mathrm{m}$ silicate feature $I_{\mathrm{si}}$ indicates the distribution of small silicate grains in the dust plume. In order to investigate the radial profile of spatial dust distributions, radial distance $r$ is multiplied to the flux to reduce the geometrical effect. This $r I_{\mathrm{si}}$ is shown in Fig. 1. The distributions at $\sim 1,2$, 3 , and 3.5 hours after the impact are shown. There is a peak in each time and that the peak moves outward. We tentatively fit each distribution with a Gaussian and obtain the peak positions. The peak positions $r_{\text {peak }}$ are plotted as a function of time (Fig. 2), showing that the peak moves linearly. The linear fit indicates a characteristic velocity of grains of $111.1 \pm 0.6 \mathrm{~m} / \mathrm{s}$. Thus, we find that the velocity of small silicate grains ( $\sim$ submicrons) is $\sim 100 \mathrm{~m} / \mathrm{s}$.

Next, we consider $I_{\text {cont }}$ (Fig. 3). Again, we multiplied $r$ with the light flux $I_{\text {cont }}$. The distributions of $r I_{\text {cont }}$ is

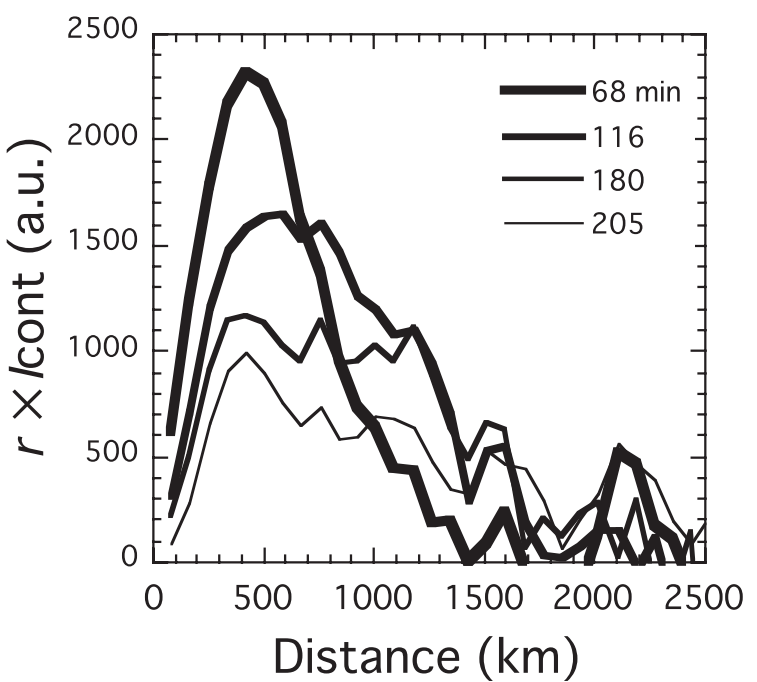

Fig. 3. Radial profiles of $I_{\text {cont }}=\left(I_{8.8}+I_{12.4}\right) / 2$ multiplied by $r$ as a function of time.

significantly different from $r I_{\mathrm{si}}$ in Fig. 1. Although the distribution profile broadens with time, the peak position of $r I_{\text {cont }}$ does not exhibit a clear outward motion. Parhaps, a more outstanding change with time is the radial spread of the distribution. The full width half maximum (FWHM) of the distribution of $r I_{\text {cont }}$ increases from $\sim 560 \mathrm{~km}$ at 1 hour to $\sim 1400 \mathrm{~km}$ at 3 hours after the impact. The continuum component $I_{\text {cont }}$ is expected to be contributed by not only small grains but also larger ones, whereas the $I_{\mathrm{si}}$ component is primarily from small grains within the DI ejecta plume. Hence, the observed difference between $r I_{\mathrm{si}}$ and $r I_{\text {cont }}$ should reflect the nature of the size distribution of grains. In the next section we estimate the size distribution of grains from the observed flux, $I_{\text {cont }}$.

\section{Discussion}

A classic ejecta curtain model predicts a monotonically decreasing mass distribution as a function of ejection velocity (e.g., Housen et al., 1983); that is, there should not be such a peak in Fig. 1. This discrepancy has been pointed out (e.g., Sugita et al., 2005) and several authors suggest that an acceleration process for dust grains in the inner portion of the plume may occur caused by volatile evaporation and expansion. Küppers et al. (2005) observed a large amount of water vapor, $\mathrm{Mg} \sim 4.6 \times 10^{6} \mathrm{~kg}$. They also suggested that this vapor accelerates solid grains. Also, analyses of both radial and angular profiles show that, though the outer part of the dust plume is conical fan-shape (this is consistent with experimental results in laboratories), the inner part is strongly influenced by the dynamics of volatile evaporation and expansion (Sugita et al., 2005).

Here, we consider the dust grains in the inner part of the plume. If the gas drag accelerates the grains ejected by the DI event, the equation of motion of the grains is,

$$
\rho_{\mathrm{s}} \frac{\pi}{6} D_{\mathrm{p}}^{3} \frac{d v_{\mathrm{s}}}{d t}=C_{\mathrm{D}} \rho_{\mathrm{g}}\left(v_{\mathrm{s}}-v_{\mathrm{g}}\right)^{2} \frac{\pi D_{\mathrm{p}}^{2}}{4},
$$

where $\rho_{\mathrm{s}}, v_{\mathrm{s}}, \rho_{\mathrm{g}}, v_{\mathrm{g}}, C_{\mathrm{D}}$, and $D_{\mathrm{p}}$ are the density and the velocity of solid grains, the gas density, the gas velocity, the 


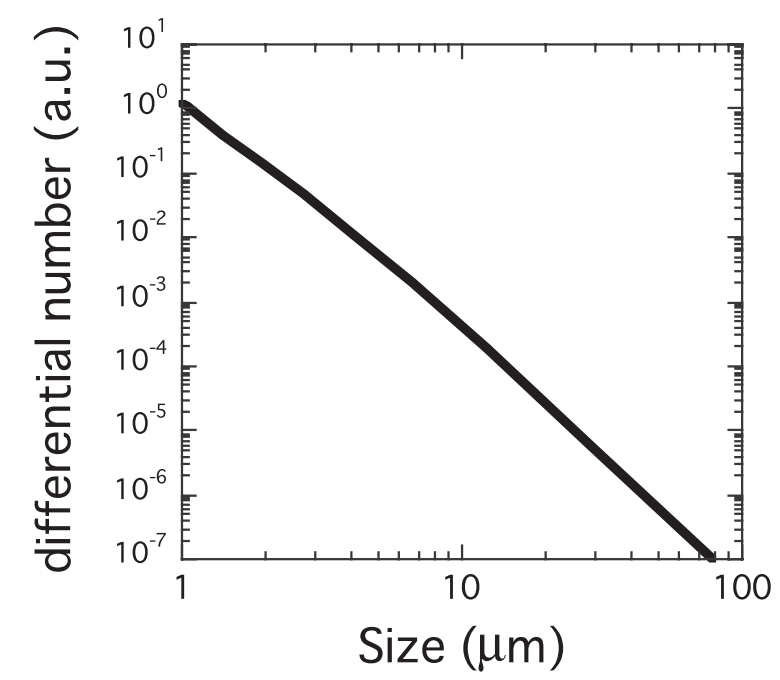

Fig. 4. The differential size distribution of ejected grains estimated from the observed flux. For simplicity, we plot only the case of 2 hours after the impact, but the results from $I_{\text {cont }}(r)$ at the other times are almost the same. At a size larger than $\sim 10 \mu \mathrm{m}$, the distribution is expressed by a power-law and the index is $\sim-4$.

drag coefficient, and the size of solid grains, respectively. The dust grains are assumed to be spherical. This equation can be solved as,

$$
\frac{3}{2} C_{\mathrm{D}} \frac{\rho_{\mathrm{g}}}{\rho_{\mathrm{s}}} \frac{r}{D_{\mathrm{p}}}=\frac{v_{\mathrm{s}}}{v_{\mathrm{g}}-v_{\mathrm{s}}}+\ln \left(1-\frac{v_{\mathrm{s}}}{v_{\mathrm{g}}}\right) .
$$

Here, $v_{\mathrm{s}}$ is obtained as a function of $r$. The terminal velocity $v_{\mathrm{sf}}$ of a grain with a size of $D_{\mathrm{p}}$ satisfies,

$$
\frac{3}{2} C_{\mathrm{D}} \frac{\rho_{\mathrm{g}}}{\rho_{\mathrm{s}}} \frac{r_{\mathrm{a}}}{D_{\mathrm{p}}}=\frac{v_{\mathrm{sf}}}{v_{\mathrm{g}}-v_{\mathrm{sf}}}+\ln \left(1-\frac{v_{\mathrm{sf}}}{v_{\mathrm{g}}}\right),
$$

where $r_{\mathrm{a}}$ is the acceleration length. If $r_{\mathrm{a}}$ is large enough, $v_{\text {sf }}$ should become $v_{\mathrm{g}}$. As shown in Fig. 2, small grains have achieved a constant velocity at our observation time ( $\sim 1$ hour after the impact), $\sim 100 \mathrm{~m} / \mathrm{s}$, which is rather smaller than the sound velocity of $\mathrm{H}_{2} \mathrm{O}$ gas at a temperature of $300 \mathrm{~K}, 430 \mathrm{~m} / \mathrm{s}$. That is, the acceleration by gas would terminate at a region nearer the nucleus where we observe. Hence, we assume that $r_{\mathrm{a}}$ is rather shorter than the region where we observe (i.e., $r_{\mathrm{a}}<\sim 100 \mathrm{~km}$ ) and that all grains have achieved terminal velocities when we observe. If we also assume that $v_{\mathrm{g}}$ is the sound velocity of $\mathrm{H}_{2} \mathrm{O}$ gas at a temperature of $300 \mathrm{~K}, 430 \mathrm{~m} / \mathrm{s}, \rho_{\mathrm{s}}, \rho_{\mathrm{g}}, C_{\mathrm{D}}$, and $r_{\mathrm{a}}$ are the same for all grains, and that $v_{\mathrm{sf}}$ is $111 \mathrm{~m} / \mathrm{s}$ when $D_{\mathrm{p}}$ is $1 \mu \mathrm{m}$ (Fig. 2), the relation between $D_{\mathrm{p}}$ and the terminal velocity $v_{\text {sf }}$ can be obtained from Eq. (3),

$$
\left(\frac{D_{\mathrm{p}}}{\mu \mathrm{m}}\right)=0.049 /\left[\frac{v_{\mathrm{sf}}}{430-v_{\mathrm{sf}}}+\ln \left(1-\frac{v_{\mathrm{sf}}}{430}\right)\right] .
$$

This equation shows that fast grains are small while slow ones are large. This is consistent with the results obtained by Harker et al. (2007) using mid-IR spectra of the impact; they also find that the smaller grains travel faster than the larger grains, in support of Eq. (4).

Since $v_{\mathrm{sf}}$ can be written as $v_{\mathrm{sf}}=r / t$ for $r>r_{\mathrm{a}}$, Eq. (4) becomes a relation between $D_{\mathrm{p}}$ and $r$ at a time $t$,

$$
\left(\frac{D_{\mathrm{p}}}{\mu \mathrm{m}}\right)=0.049 /\left[\frac{r}{430 t-r}+\ln \left(1-\frac{r}{430 t}\right)\right] .
$$

This equation gives the mean radial position of ejecta grains as a function of time and size.

Observed flux is obtained as a function of $r, I_{\text {cont }}(r) d r$. Using Eq. (5), this can be written as a function $D_{\mathrm{p}}$, $I_{\text {cont }}\left(D_{\mathrm{p}}\right)\left(d r / d D_{\mathrm{p}}\right) d D_{\mathrm{p}}$. When the size-number distribution is $n\left(D_{\mathrm{p}}\right) d D_{\mathrm{p}}$, we assume that the observed flux is proportional to the total cross section of grains, that is, $I_{\text {cont }}\left(D_{\mathrm{p}}\right)\left(d r / d D_{\mathrm{p}}\right) d D_{\mathrm{p}} \sim D_{\mathrm{p}}^{2} n\left(D_{\mathrm{p}}\right) d D_{\mathrm{p}}$. This becomes $n\left(D_{\mathrm{p}}\right) \sim I_{\text {cont }}\left(D_{\mathrm{p}}\right)\left(d r / d D_{\mathrm{p}}\right) / D_{\mathrm{p}}^{2}$. Thus, $n\left(D_{\mathrm{p}}\right)$ is obtained as a function of $D_{\mathrm{p}}$. A result is shown in Fig. 4. For simplicity, we plot only the case of 2 hours after the impact, but the results from $I_{\text {cont }}(r)$ at the other times are almost the same. The obtained distribution for grains larger than $\sim 10 \mu \mathrm{m}$ is fit well by a power-law, $n\left(D_{\mathrm{p}}\right) \sim D_{\mathrm{p}}^{-\alpha}$ and the index $\alpha$ is $\sim 4$, which is slightly larger than the values provided by fitting the observed spectrum with a power-law in the large grain size range (from $<1$ to $\sim 10 \mu \mathrm{m}$ across), an exponent of between -3.5 and -3.6 (Sugita et al., 2005; Ootsubo et al., 2007) and -3.7 (Harker et al., 2007).

\section{Summary}

We propose an alternative method to estimate the size distribution at a size larger than $\sim 10 \mu \mathrm{m}$ based on the observational data of the DI ejecta in both time and space. We consider the radial distributions of $I_{\mathrm{si}}$ and $I_{\text {cont }}$ at P.A. 225. The distribution of $I_{\text {si }}$ has a peak which moves $\sim 100 \mathrm{~m} / \mathrm{s}$. Assuming that the evaporation of volatiles occurs and that the expansion of the vapor accelerates solid dust grains, we obtain an analytical relation between $D_{\mathrm{p}}$ and $r$. Based on this relation and the observational result of $I_{\text {cont }}$, the sizenumber distribution of larger grains is estimated. The result indicates that the distribution is expressed as a power law with an exponent $\sim-4$.

Because the contribution of the large grains to the total ejecta mass has been highly uncertain and spectral analyses using the data at the mid-infrared wavelength do not clearly indicate the size distribution at a size larger than $\sim 10 \mu \mathrm{m}$, such an estimate on their size distribution is of great importance, though detailed analyses and numerical simulations should be necessary to evaluate the error of this method.

Finally, it is noted that the analysis given by Kadono et al. (2007) is on the outer part of the ejecta plume, where the effect of gas acceleration is minimal. The present study focuses on the inner part of the ejecta, where the gas acceleration effect can be significant. In this sense, these two studies are complimentary to each other.

Acknowledgments. This paper is based on data collected at the Subaru Telescope, which is operated by the National Astronomical Observatory of Japan (S05A-042 and S05A-OT11). The authors are grateful to two anonymous reviewers for useful comments. This research is partially supported by Grant in Aid from the Japan Society for the Promotion of Science.

\section{References}

A'Hearn, M. F. et al., Deep Impact: Excavating comet Tempel 1, Science, 310, 258-264, 2005. 
Harker, D. E., C. E. Woodward, D. H. Wooden, R. S. Fisher, and C. A. Trujillo, Gemini-N mid-IR observations of the dust properties of the ejecta excavated from Comet 9P/Tempel 1 during Deep Impact, Icarus, 190, 432-453, 2007

Housen, K. R., R. M. Schmidt, and K. A. Holsapple, Crater ejecta scaling laws-Fundamental forms based on dimensional analysis, J. Geophys. Res., 88, 2485-2499, 1983.

Jorda, L., P. Lamy, G. Faury, H. U. Keller, S. Hviid, M. Küppers, D. Koschny, J. Lecacheux, P. Guiérrez, and L. M. Lara, Properties of the dust cloud caused by the Deep Impact experiment, Icarus, 187, 208219, 2007.

Kadono, T., S. Sugita, S. Sako, T. Ootsubo, M. Honda, H. Kawakita, T. Miyata, R. Furusho, and J. Watanabe, The thickness and formation age of the surface layer on comet 9P/Tempel 1, Astrophys. J. Lett., 661, L89L92, 2007.

Küppers, M. et al., A large dust/ice ratio in the nucleus of comet
9P/Tempel 1, Nature, 437, 987-990, 2005.

Melosh, H. J., Impact Cratering: A Geologic Process, 245 pp., Oxford Univ. Press, New York, 1989.

Ootsubo, T., S. Sugita, T. Kadono, M. Honda, I. Sakon, H. Kawakita, J. Watanabe, and Subaru/COMICS team, Mid-Infrared observation of the dust plume from comet 9P/Tempel 1 generated by the Deep Impact collision using Subaru/COMICS, in the Proc. Dust in Planet. Sys., Workshop, September 26-30, 2005, Kauai, Hawai'i, edited by H. Kruger and A. Graps, ESA Publications, SP-643, 45-48, 2007.

Sugita, S. et al., Subaru telescope observations of Deep Impact, Science, 310, 274-278, 2005.

T. Kadono (e-mail: kadonot@ile.osaka-u.ac.jp), S. Sugita, T. Ootsubo, S. Sako, T. Miyata, R. Furusho, M. Honda, H. Kawakita, and J. Watanabe 